\title{
Aportes a la flora polínica de turberas altoandinas, Provincia de Jujuy, noroeste argentino
}

\section{Contributions to the pollen flora of high-Andean cushion peatlands, Jujuy Province, Northwestern Argentina}

\author{
Gonzalo R. Torres ${ }^{1,2}$, Liliana C. Lupo ${ }^{1,2}$, Ana C. Sánchez ${ }^{1,3}$ \& Karsten Schittek ${ }^{4}$ \\ ${ }^{1}$ Laboratorio de Palinología - Facultad de Ciencias Agrarias. Universidad Nacional de Jujuy. Alberdi 47, 4600, San Salvador \\ de Jujuy, Argentina. \\ ${ }^{2}$ CONICET. \\ ${ }^{3}$ Cátedra de Botánica Sistemática y Fitogeografía - Facultad de Ciencias Agrarias. Universidad Nacional de Jujuy. Alberdi 47, \\ 4600, San Salvador de Jujuy, Argentina. \\ ${ }^{4}$ Geographisches Institut, Universität Heidelberg. Im Neuenheimer Feld 348. 69120 Heidelberg. Alemania. \\ gztorres@gmail.com
}

\begin{abstract}
RESUMEN
Se presenta la caracterización polínica de 15 especies, nuevas para la palinoflora andina, distribuidas en 12 familias, con una clave para su identificación. Las mismas pertenecen a la flora actual de comunidades vegetales de turberas altoandinas. Estos estudios tienen como objetivo aportar al conocimiento polínico de los ambientes de turberas altoandinas, con el fin de optimizar la identificación de tipos polínicos presentes en los registros fósiles del cuaternario de la región del noroeste argentino (NOA). Las descripciones pertenecen a las siguientes especies: Apiaceae: Lilaeopsis macloviana, Asteraceae: Cuatrecasasiella argentina, Perezia pygmaea, Brassicaceae: Eudema friesii, Cyperaceae: Carex gayana, Zameioscirpus muticus, Fabaceae: Astragalus micranthellus, Gentianaceae: Gentianella meyeniana, Juncaceae: Distichia muscoides, Orobanchaceae: Bartsia crenoloba, Castilleja pumila, Plantaginaceae: Plantago tubulosa, Poaceae: Puccinellia frigida, Portulacaceae: Calandrinia compacta, Ranunculaceae: Halerpestes exilis.
\end{abstract}

Palabras clave: Palinología, turberas, Andes centrales, noroeste argentino.

\begin{abstract}
The descriptions of 15 pollen types, new for the high-Andean pollen flora, distributed in 12 families, are presented, with a key for their identification. They belong to the current flora of cushion peatlands' plants communities. The aim of these studies is to contribute to the knowledge of pollen of high Andean peatland ecosystems, in order to optimize the identification of pollen types conserved in quaternary fossil records in Northwestern Argentina (NOA). The following species are described: Apiaceae: Lilaeopsis macloviana, Asteraceae: Cuatrecasasiella argentina, Perezia pygmaea, Brassicaceae: Eudema friesii, Cyperaceae: Carex gayana, Zameioscirpus muticus, Fabaceae: Astragalus micranthellus, Gentianaceae: Gentianella meyeniana, Juncaceae: Distichia muscoides, Orobanchaceae: Bartsia crenoloba, Castilleja pumila, Plantaginaceae: Plantago tubulosa, Poaceae: Puccinellia frigida, Portulacaceae: Calandrinia compacta, Ranunculaceae: Halerpestes exilis.
\end{abstract}

KEYwORDs: Palynology, peatlands, central Andes, Northwestern Argentina.

\section{INTRODUCCIÓN}

Las turberas altoandinas o "bofedales" se forman entre los 4.000 y 4.800 m s.n.m. en las altas cumbres, ubicadas en pendientes o en el llano y se distribuyen en forma dispersa en las ecoregiones del piso puneño y altoandino. Estos hábitats naturales presentan aportes de humedad permanente, ya que se alimentan de manantiales y cursos de agua del escalón altoandino. Están conformadas por comunidades vegetales palustres y semiacuáticas, con dominancia de plantas de estructura almohadillada, principalmente las juncáceas Distichia muscoides Nees \& Meyen y Oxychloë andina Phil. (Squeo et al. 2006).

Su composición florística varía dependiendo de factores 
ecológicos naturales (condiciones climáticas, calidad y cantidad del abastecimiento de agua, salinidad, etc.), por lo que albergan una gran diversidad, con especies exclusivas, que a su vez tienen un rol clave en la regulación del balance hídrico regional (Torres et al. 2007). Ruthsatz (2008) ha demostrado que la variación florística y ecológica de las turberas altoandinas del noroeste argentino reflejan claramente un marcado gradiente climático desde el noreste semi-húmedo al suroeste sub-árido de la zona. De acuerdo al contexto descripto, las turberas altoandinas pueden considerarse buenos indicadores de cambios de condiciones climáticas, especialmente de variaciones de humedad y temperatura. Por otra parte, estos ecosistemas son considerados frágiles y su valor biológico reside en las variadas y originales comunidades de especies y endemismos que albergan (Hails 1996, Ramsar 2004). No obstante, estas comunidades siempreverdes revisten gran importancia para los herbívoros, nativos y domésticos de la región, por lo que el sobrepastoreo constituye la principal problemática de degradación, puesto que reduce la cobertura vegetal, dejando al suelo muy susceptible a la erosión y causa un cambio en la composición botánica original (Braun Wilke \& Picchetti 1999).

La formación de turba y su lenta descomposición, asociado a la acumulación de profundos estratos de material orgánico favorecen la preservación del polen en el tiempo. Considerando que, desde un punto de vista paleoecológico, las turberas altoandinas han integrado el paisaje andino, del noroeste argentino, desde el Cuaternario Superior, es posible obtener un archivo acerca de variaciones paleoambientales, factible de correlacionar con modelos generales de cambio climático para el pasado reciente (Earle et al. 2003). Sin embargo, las investigaciones paleoecológicas se ven limitadas por la escasa información polínica de ambientes locales de turberas que faciliten la identificación de especies fósiles presentes en sedimentos cuaternarios de la zona.

Diferentes autores han abordado estudios de morfología polínica de diversas regiones, en las cuales se incluyen especies de turberas altoandinas. En todos los casos son atlas generales o estudios polínicos de sedimentos. Se destacan los trabajos para Chile (Heusser 1971), de Argentina (Markgraf \& D'Antoni 1978, Wingenroth \& Heusser 1984) y Bolivia (Graf 1979, 1992). El presente trabajo tiene como objetivo aportar al conocimiento polínico de los ambientes de turberas altoandinas, con el fin de optimizar la identificación de tipos polínicos presentes en los registros fósiles del cuaternario de la región del noroeste argentino (NOA).

\section{MATERIALES Y MÉTODOS}

El área estudiada abarca sectores del Borde Oriental y Occidental de Puna del noroeste argentino (entre $22 \mathrm{~N} \mathrm{y}$ $26 \mathrm{~N}, 65 \mathrm{~W}$ y $67 \mathrm{~W}$ ) y pertenece fitogeográficamente a la Provincia Altoandina (Cabrera 1976).
El material proviene de diferentes turberas (Fig. 1), las cuales fueron visitadas en distintas campañas. Se utilizó el material actual en flor, el cual fue recolectado siguiendo la metodología estándar de herborización. La identificación de las especies vegetales se realizó en terreno y en laboratorio, como también se contó con la colaboración de especialistas y literatura taxonómica pertinente.

La técnica aplicada para el procesamiento de las flores fue la de Erdtman (1960), consistente en un tratamiento con hidróxido de potasio al $10 \%$ con posterior acetólisis; el montado se efectuó con glicerina y el sellado se realizó con parafina. Las muestras fueron depositadas en la Palinoteca del Laboratorio de Palinología de la Facultad de Ciencias Agrarias de la Universidad Nacional de Jujuy (PAL-JUA).

Para la caracterización morfológica de los granos de polen se realizaron observaciones con microscopio óptico. Se describió y midió en 100X. Para cada carácter estudiado se menciona la dimensión mínima y máxima encontradas para el diámetro ecuatorial (DE), eje polar (EP), excluida la exina, espesor de la exina, diferenciándose entre sexina y nexina en los casos observables, largo de espinas, diámetro de poros u ora según corresponda, longitud de los colpos siempre que sus extremos sean visibles en vista ecuatorial (de no ser así se indica colpos largos), apocolpio y mesocolpio. Se tomaron como mínimo 25 mediciones. Las fotos fueron tomadas con cámaras de microscopios digitales Leica ICC50 (Universidad Nacional de Jujuy, Argentina) y Zeiss Axio Imagen 2 (Universität Trier, Alemania).

La terminología utilizada en la descripción polínica fue tomada de Erdtman (1957), Faegri \& Iversen (1989), Moore et al. (1991) y Punt et al. (2007). La nomenclatura de las plantas sigue a Zuloaga et al. (2008).

\section{RESULTADOS}

Se presenta la caracterización polínica de 15 especies, distribuidas en 12 familias, con una clave para su identificación.

\section{Apiaceae - Lilaeopsis macloviana (Gand.) A.W.Hill}

Fig. 2 A-B.

Granos tricolporados, prolados. Ámbito circular. Diámetro ecuatorial de 18 a $25 \mu \mathrm{m}$, eje polar de 27 a $35 \mu \mathrm{m}$. Colpos largos. Apocolpio de 5 a $8 \mu \mathrm{m}$, mesocolpio de 10 a 15 $\mu \mathrm{m}$. Ora circular de $3 \mu \mathrm{m}$ de diámetro. Exina semitectada, microreticulada de $2 \mu \mathrm{m}$ de espesor.

Material estudiado: ARGentinA. Prov. Jujuy. Dpto. Humahuaca. Laguna Leandro. 4.050 m. 06-II-2007. Schittek V0380705 (PAL-JUA N ${ }^{\circ}$ GT 046).

2. Asteraceae - Cuatrecasasiella argentina (Cabrera) H.Rob.

Fig. 2 C-D. 
Granos tricolporados, suboblados a oblado esferoidales. Ámbito circular. Diámetro ecuatorial de 24 a $25 \mu \mathrm{m}$, eje polar de 21 a $27 \mu \mathrm{m}$. Colpos largos. Apocolpio de 4 a 6 $\mu \mathrm{m}$, mesocolpio de 15 a $18 \mu \mathrm{m}$. Ora circular de $4 \mu \mathrm{m}$ de diámetro. Exina tectada, equinada, de 2 a $3 \mu \mathrm{m}$ de espesor (sexina de 1 a $2 \mu \mathrm{m}$, nexina de $1 \mu \mathrm{m}$ ). Espinas de $3 \mu \mathrm{m}$ de alto.

Material estudiado: ARGentinA. Prov. Jujuy. Dpto. Humahuaca. Aparzo. 4.200 m. 2-III-2006. Schittek V0020605 (PAL-JUA Nº GT 048).

\section{Asteraceae - Perezia pygmaea Wedd.}

Fig. 2 E-F.

Granos tricolporados, prolado esferoidales. Ámbito circular. Diámetro ecuatorial de 35 a $47 \mu \mathrm{m}$, eje polar de 40 a 45 $\mu \mathrm{m}$. Colpos largos. Apocolpio de 3 a $5 \mu \mathrm{m}$, mesocolpio de 20 a $30 \mu \mathrm{m}$. Ora lalongado de 15 por $5 \mu \mathrm{m}$. Exina tectada, microequinada, de 3 a $4 \mu \mathrm{m}$ de espesor (sexina de 2 a $3 \mu \mathrm{m}$, nexina de $1 \mu \mathrm{m}$ ), adelgazándose hacia los colpos y hacia los polos. Espínulas de $<1 \mu \mathrm{m}$ de alto.

Material estudiado: ARGentinA. Prov. Jujuy. Dpto. Humahuaca. Laguna Verde. 4.200 m. 25-III-2006. Torres 004 (PAL-JUA N ${ }^{\circ}$ GT 004).

\section{Brassicaceae - Eudema friesii O.E.Schulz}

Fig. 2 G-H.

Granos tricolpados, prolados esferoidales. Ámbito subtriangular a circular. Diámetro ecuatorial de 20 a 27 $\mu \mathrm{m}$, eje polar de 23 a $28 \mu \mathrm{m}$. Colpos largos. Apocolpio de 7 a $9 \mu \mathrm{m}$, mesocolpio de 15 a $17 \mu \mathrm{m}$. Exina semitectada, reticulada, heterobrochada, de 3 a $4 \mu \mathrm{m}$ de espesor. Los brochis disminuyen hacia los colpos.

Material estudiado: ARGentinA. Prov. Jujuy. Dpto. Susques. Coranzuli. 4.100 m. 10-III-2006. Schittek V0190601 (PAL-JUA Nº GT 017 ).

\section{Cyperaceae - Carex gayana E.Desv.}

Fig. 2 I.

Granos periporados, tetraédricos de lados convexos. Diámetro ecuatorial de 28 a $30 \mu \mathrm{m}$, eje polar de 32 a 35 $\mu \mathrm{m}$. Cuatro poros circulares de $5 \mu \mathrm{m}$ de diámetro dispuestos lateralmente y un poro basal. Exina tectada perforada de 1-2 $\mu \mathrm{m}$ de espesor, disgregándose hacia los poros.

Material estudiado: ARGENTINA. Prov. Jujuy. Dpto. Cochinoca. Cochinoca. 3.900 m. 05-II-2007. Torres 035 (PAL-JUA N ${ }^{\circ}$ GT 035).

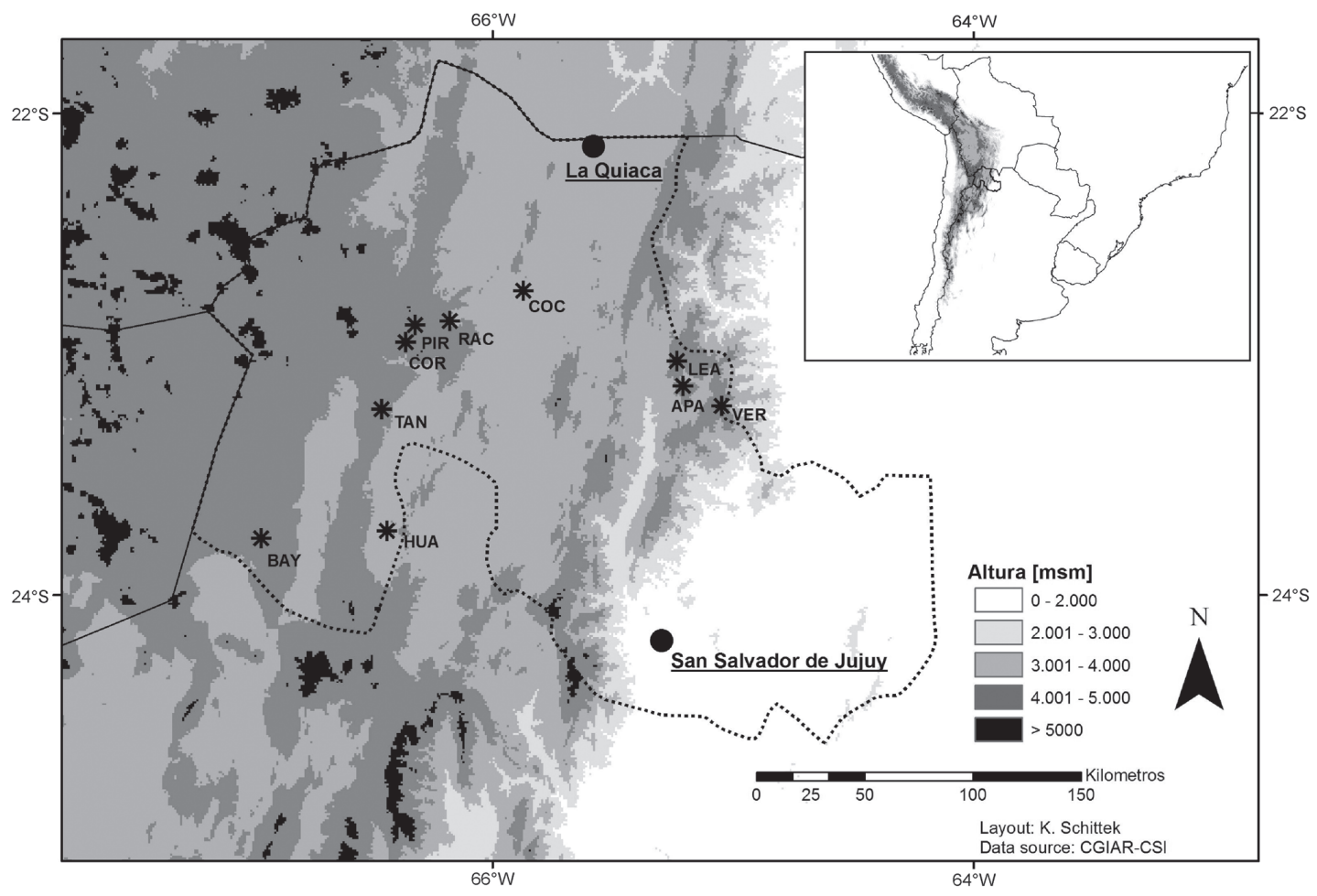

Figura 1. Ubicación de las turberas altoandinas estudiadas. Aparzo (APA), Cochinoca (COC), Coranzuli (COR), Huancar (HUA), Laguna Leandro (LEA), Laguna Verde (VER), Los Bayos (BAY), Mina Pirquitas (PIR), Rachaite (RAC), Tanques (TAN).

Figure 1. Location of the investigated high-Andean peatlands. Aparzo (APA), Cochinoca (COC), Coranzuli (COR), Huancar (HUA), Laguna Leandro (LEA), Laguna Verde (VER), Los Bayos (BAY), Mina Pirquitas (PIR), Rachaite (RAC), Tanques (TAN). 

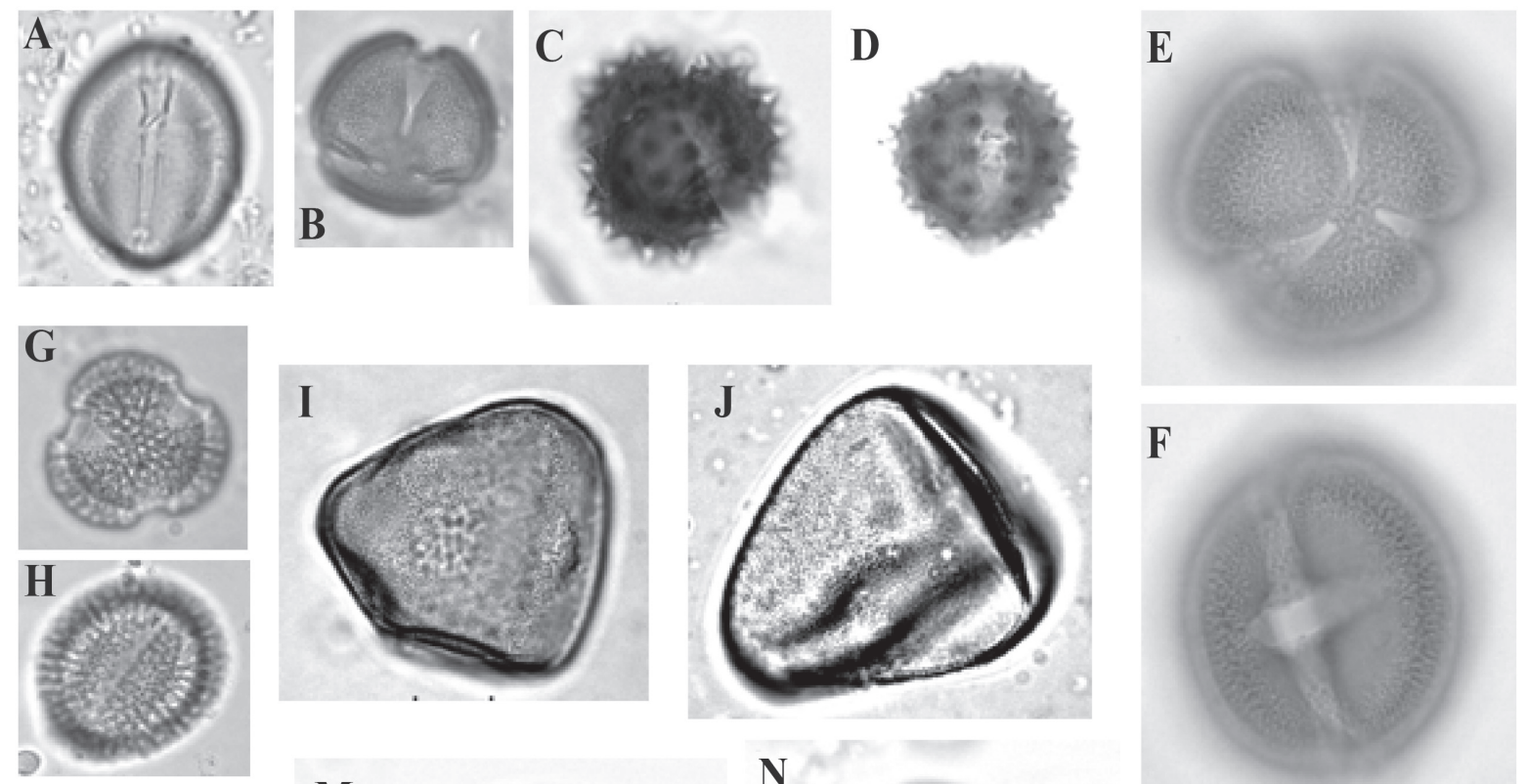

F
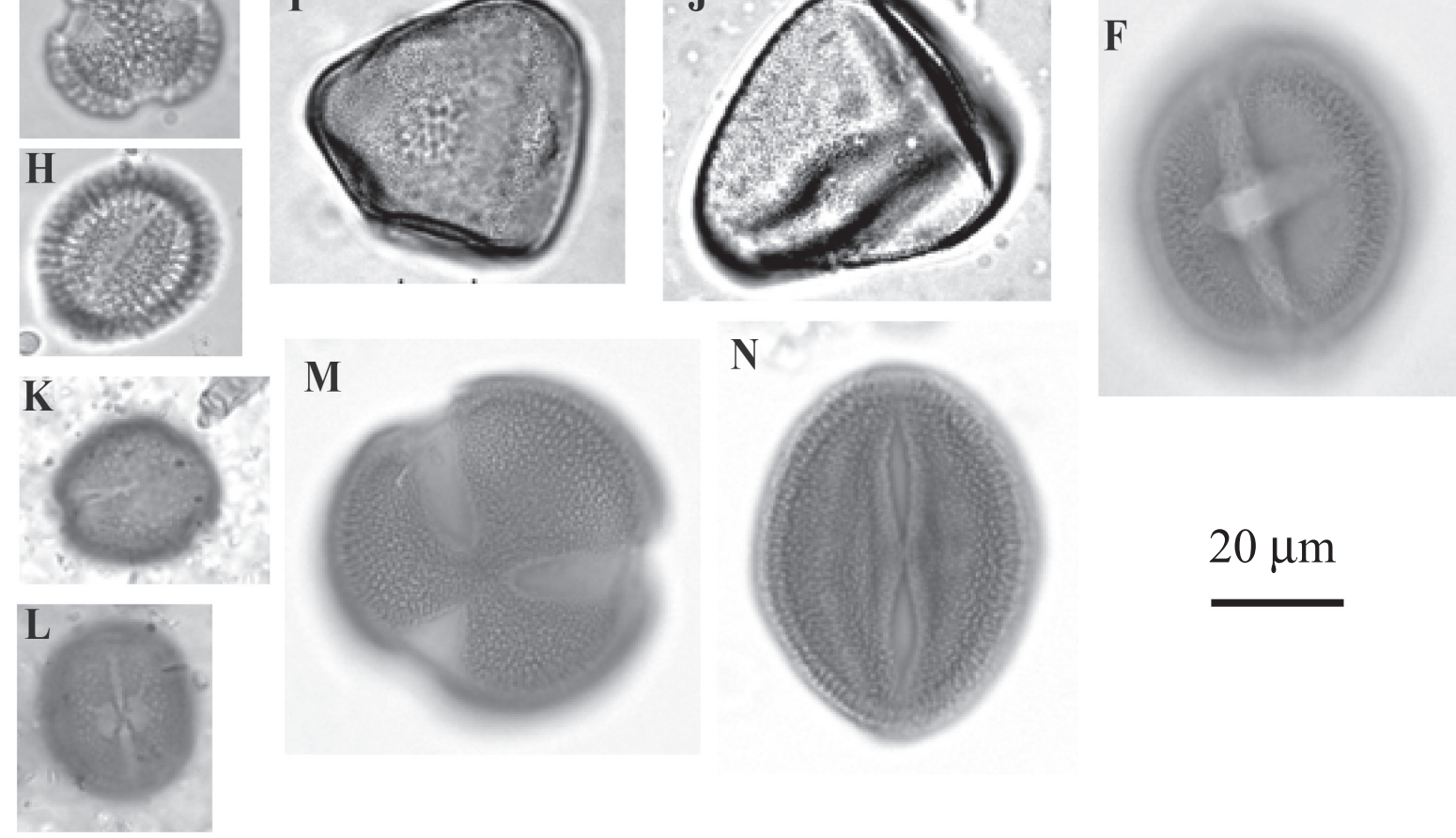

Figura 2. Tipos polínicos descriptos. A-B: Lilaeopsis macloviana, A: Vista ecuatorial, B: Vista polar. C-D: Cuatrecasasiella argentina, C: Vista polar, D: Vista ecuatorial. E-F: Perezia pygmaea, E: Vista polar, F: Vista ecuatorial. G-H: Eudema friesii, G: Vista polar, H: Vista ecuatorial. I: Carex gayana vista general. J: Zameioscirpus muticus vista general. K-L: Astragalus micranthellus, K: Vista polar, L: Vista ecuatorial. M-N: Gentianella meyeniana, M: Vista polar, N: Vista ecuatorial.

Figure 2. Pollen types descripted. A-B: Lilaeopsis macloviana, A: Ecuatorial view, B: Polar view. C-D: Cuatrecasasiella argentina, C: Polar view, D: Ecuatorial view. E-F: Perezia pygmaea, E: Polar view, F: Ecuatorial view. G-H: Eudema friesii, G: Polar view, H: Ecuatorial view. I: Carex gayana, general view. J: Zameioscirpus muticus, general view. K-L: Astragalus micranthellus, K: Polar view, L: Ecuatorial view. M-N: Gentianella meyeniana, M: Polar view, N: Ecuatorial view.

\section{Cyperaceae - Zameioscirpus muticus Dhooge \& Goetgh.}

Fig. $2 \mathrm{~J}$.

Granos periporados, tetraédricos de lados convexos. Diámetro ecuatorial de 35 a $40 \mu \mathrm{m}$, eje polar de 40 a 45 $\mu \mathrm{m}$. Cuatro poros de 8 a $10 \mu \mathrm{m}$ de diámetro de márgenes irregulares, dispuestos lateralmente y un poro basal. Exina tectada perforada de $2 \mu \mathrm{m}$ de espesor (sexina de $1 \mu \mathrm{m}$, nexina de $1 \mu \mathrm{m}$ ), disgregándose hacia los poros.

Material estudiado: ARGentinA. Prov. Jujuy. Dpto. Humahuaca. Aparzo. 4.100 m. 22-III-2006. Torres 016 (PAL-JUA N ${ }^{\circ}$ GT 016).

\section{Fabaceae - Astragalus micranthellus Wedd.}

Fig. 2 K-L.

Granos tricolporados, subprolados. Ámbito circular. Diámetro ecuatorial de 20 a $24 \mu \mathrm{m}$, eje polar de 25 a 29 $\mu \mathrm{m}$. Colpos de $19 \mu \mathrm{m}$ de largo. Apocolpio de 9 a $11 \mu \mathrm{m}$, mesocolpio de 15 a $17 \mu \mathrm{m}$. Ora lalongado de 6 por $4 \mu \mathrm{m}$. Exina semitectada, reticulada, heterobrochada, de $2 \mu \mathrm{m}$ de espesor. Los brochi disminuyen hacia los colpos.

Material estudiado: ARGEnTinA. Prov. Jujuy. Dpto. Rinconada. Mina Pirquitas. 4.300 m. 26-III-2006. Schittek V0250601 (PAL-JUA Nº GT 009). 
8. Gentianaceae - Gentianella meyeniana (Griseb.) Fabris Fig. 2 M-N.

Granos tricolporados, prolados. Ámbito circular. Diámetro ecuatorial de 30 a $40 \mu \mathrm{m}$, eje polar de 40 a $55 \mu \mathrm{m}$. Colpos largos, con constricción ecuatorial. Apocolpio de 8 a 12 $\mu \mathrm{m}$, mesocolpio de 20 a $25 \mu \mathrm{m}$. Ora circular de $5 \mu \mathrm{m}$ de diámetro a lolongado de $10-12$ por $3 \mu \mathrm{m}$, de margenes irregulares. Exina semitectada, reticulada, de 3-4 $\mu \mathrm{m}$ de grosor (sexina de 2-3 $\mu \mathrm{m}$, nexina de $1 \mu \mathrm{m}$ ), adelgazándose hacia los colpos.

Material estudiado: ARGentinA. Prov. Jujuy. Dpto.
Susques. Tanques. 8-II-2007. 4.200 m. Schittek V0400708 (PAL-JUA N ${ }^{\circ}$ GT 020).

\section{Juncaceae - Distichia muscoides Nees \& Meyen}

Fig. 3 A.

Granos de polen dispuestos en tétrades tetraédricas de 50 a $52 \mu \mathrm{m}$ de diámetro. Mónades monoporadas. Poros de $23 \mu \mathrm{m}$ de diámetro de márgenes irregulares. Exina tectada, psilada, de $2 \mu \mathrm{m}$ de espesor, disgregándose hacia los poros.

Material estudiado: ARGEnTinA. Prov. Jujuy. Dpto. Humahuaca. Aparzo. 4.200 m. 30-X-2007. Schittek V0020708 (PAL-JUA N ${ }^{\circ}$ GT 050).
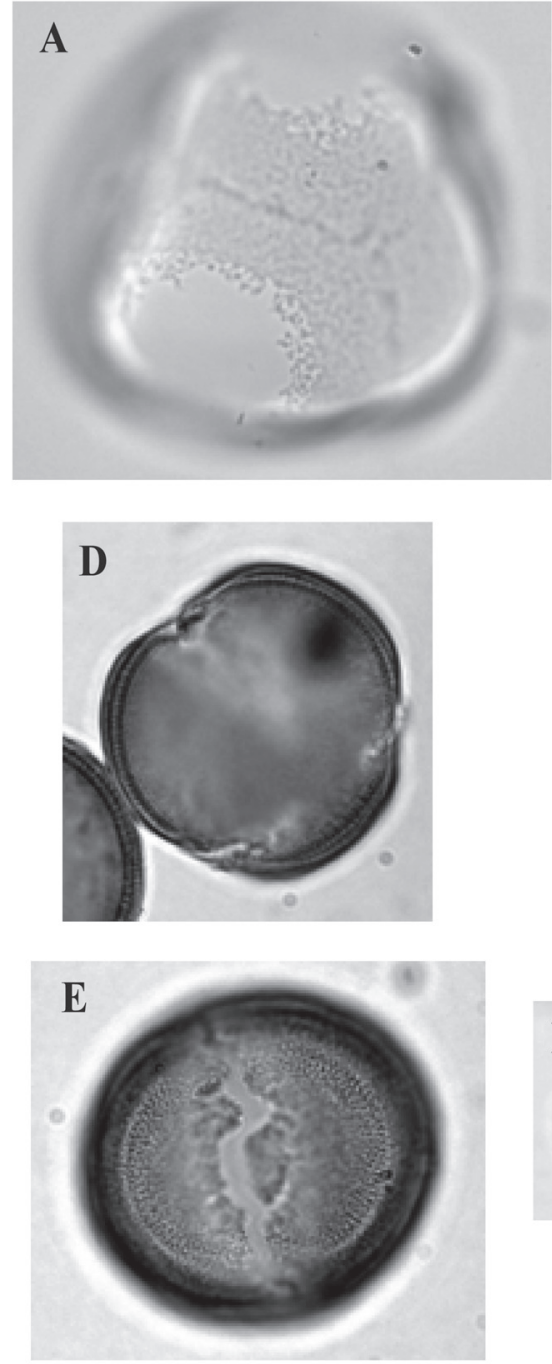

\section{B}
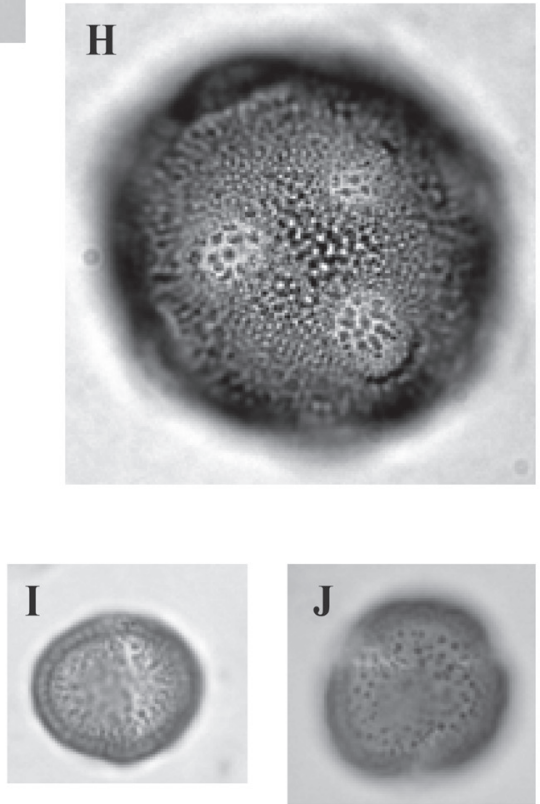
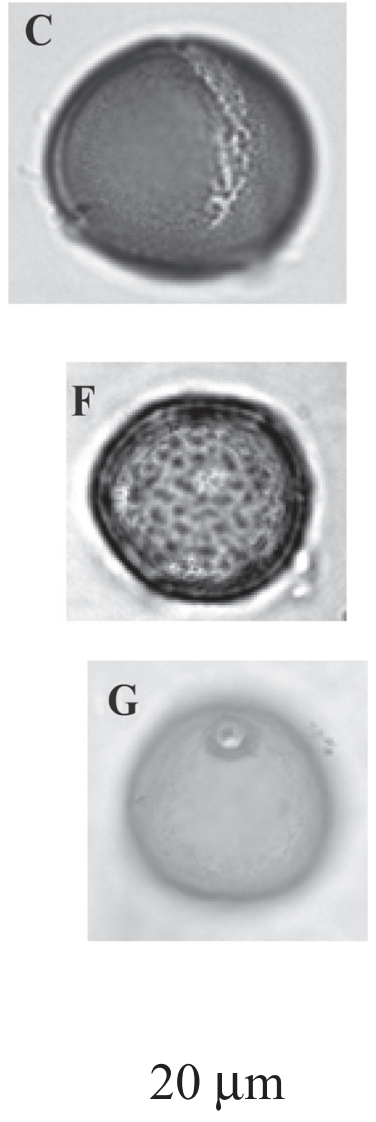

Figura 3. Tipos polínicos descriptos (continuación). A: Distichia muscoides vista general. B-C: Bartsia crenoloba, B: Vista polar, C: Vista ecuatorial. D-E: Castilleja pumila, D: Vista polar, E: Vista ecuatorial. F: Plantago tubulosa. G: Puccinellia frigida. H: Calandrinia compacta. I-J: Halerpestes exilis, I: Vista ecuatorial, J: Vista polar.

Figure 3. Pollen types descripted (continuation). A: Distichia muscoides general view. B-C: Bartsia crenoloba, B: Polar view, C: Ecuatorial view. D-E: Castilleja pumila, D: Polar view, E: Ecuatorial view. F: Plantago tubulosa. G: Puccinellia frigida. H: Calandrinia compacta. I-J: Halerpestes exilis, I: Ecuatorial view, J: Polar view. 
10. Orobanchaceae - Bartsia crenoloba Wedd.

Fig. 3 B-C.

Granos tricolpados, prolado esferoidales. Ámbito circular. Diámetro ecuatorial de 30 a $47 \mu \mathrm{m}$, eje polar de 32 a 55 $\mu \mathrm{m}$. Colpos largos de márgenes irregulares. Apocolpio de 10 a $17 \mu \mathrm{m}$, mesocolpio de 22 a $35 \mu \mathrm{m}$. Exina semitectada, microreticulada, de $2 \mu \mathrm{m}$ de grosor.

Material estudiado: ARGEnTINA. Prov. Jujuy. Dpto. Humahuaca. Laguna Verde. 4.200 m. 25-III-2006. Torres 005 (PAL-JUA N ${ }^{\circ}$ GT 005).

11. Orobanchaceae - Castilleja pumila Wedd.

Fig. 3 D-E.

Granos tricolpados, prolados esferoidales. Ámbito circular. Diámetro ecuatorial de 28 a $45 \mu \mathrm{m}$, eje polar de 32 a 49 $\mu \mathrm{m}$. Colpos largos de márgenes irregulares. Apocolpio de 10 a $15 \mu \mathrm{m}$, mesocolpio de 22 a $32 \mu \mathrm{m}$. Exina semitectada, microreticulada, de 2 a $3 \mu \mathrm{m}$ de espesor.

Material estudiado: ARGEnTINA. Prov. Jujuy. Dpto. Humahuaca. Aparzo. 4.200 m. 02-III-2006. Schittek V0010601 (PAL-JUA Nº GT 007).

\section{Plantaginaceae - Plantago tubulosa Decne.}

Fig. $3 \mathrm{~F}$.

Granos periporados, esferoidales. Ámbito circular. Diámetro de 24 a $29 \mu \mathrm{m}$. Poros de 8 a 10, circulares de $5 \mu \mathrm{m}$ de diámetro, con membrana granulada. Annulus indistinto. Exina semitectada, reticulada, de 2 a $3 \mu \mathrm{m}$ de espesor.

Material estudiado: ARGEnTINA. Prov. Jujuy. Dpto.

Rinconada. Coranzuli. 4.100 m. 08-II-2007. Torres 029

(PAL-JUA N ${ }^{\circ}$ GT 029).
13. Poaceae - Puccinellia frigida (Phil.) I.M.Johnst.

Fig. 3 G.

Granos monoporados, oblados esferoidales. Ámbito circular. Diámetro ecuatorial de 25 a $28 \mu \mathrm{m}$, eje polar de 20 a 25 $\mu \mathrm{m}$. Poro de 3 a $4 \mu \mathrm{m}$ de diámetro con annulus de $3 \mu \mathrm{m}$ de grosor. Exina tectada, escabrada, de $2 \mu \mathrm{m}$ de espesor.

Material estudiado: ARGEnTINA. Prov. Jujuy. Dpto. Susques. Huancar. 3700. 09-II-2007. Torres 037 (PAL-JUA $\mathrm{N}^{\circ}$ GT 037).

\section{Portulacaceae - Calandrinia compacta Barnéoud}

Fig. $3 \mathrm{H}$.

Granos periporados, esferoidales. Ámbito circular. Diámetro de 50 a $85 \mu \mathrm{m}$, Poros de 10 a 12, circulares a elípticos. Cuando elíptico de 8-14 por 5-10 $\mu \mathrm{m}$. A veces con membrana escasamente equinada. Exina tectada perforada, microequinada, de 3 a $4 \mu \mathrm{m}$ de espesor. Espínulas de $<1 \mu \mathrm{m}$.

Material estudiado: ARGEnTINA. Prov. Jujuy. Dpto. Susques. Los Bayos. 4.400 m. 08-III-2007. Schittek V0460702 (PAL-JUA N GT 038).

\section{Ranunculaceae - Halerpestes exilis (Phil.) Tamura}

Fig. 3 I-J.

Granos tricolpados, suboblados. Ámbito circular. Diámetro ecuatorial de 17 a $27 \mu \mathrm{m}$, eje polar de 18 a $20 \mu \mathrm{m}$. Colpos de $11 \mu \mathrm{m}$ de largo. Apocolipo de 5 a $10 \mu \mathrm{m}$, mesocolpio de 15 a $18 \mu \mathrm{m}$. Exina tectada, equinada, de $3 \mu \mathrm{m}$ de espesor (sexina de $2 \mu \mathrm{m}$, nexina de $1 \mu \mathrm{m})$. Espinas de $1 \mu \mathrm{m}$ de alto.

Material estudiado: ARGEnTINA. Prov. Jujuy. Dpto. Susques. Huancar. 3.700 m. 09-II-2007. Schittek V0420704 (PAL-JUA No GT 042).

ClaVe PARA LA IDENTIFICACIÓN DE LAS ESPECIES

1. Tétrades

Distichia muscoides

1'. Mónades.

2. Granos porados.

3. Granos monoporados

3'. Granos periporados.

4. Granos Tetraédricos de lados convexos. Exina perforada.

5. DE 28-30 $\mu \mathrm{m}$. EP 32-35 $\mu \mathrm{m}$. Diámetro de poro de $5 \mu \mathrm{m}$ Carex gayana

5'. DE 35-40 Mm. EP 40-35 $\mu \mathrm{m}$. Diámetro de poro de $8-10 \mu \mathrm{m}$

4'. Granos esferoidales.

6. Diámetro de 24 a $29 \mu \mathrm{m}$. Exina reticulada

6'. Diámetro de 50 a $85 \mu \mathrm{m}$. Exina microequinada

Puccinellia frigida

os colpados o colporados.

7. Granos colpados.

8. Exina equinada. Colpos cortos

8'. Exina reticulada. Colpos largos.

9. DE 20-27 $\mu \mathrm{m}$. EP 23-28 $\mu \mathrm{m}$. Exina de 3 a $4 \mu \mathrm{m}$ de espesor ......................................................................... Eudema friesii

9'. DE 28-47 $\mu \mathrm{m}$. EP 32-55 $\mu \mathrm{m}$. Exina de 2 a $3 \mu \mathrm{m}$ de espesor, microreticulada ..........Bartsia crenoloba y Castilleja pumila

7'. Granos colporados.

10. Suboblados a oblado esferoidales. Exina equinada

10'. Granos prolados. Exina variable.

11. Ora circular de $3 \mu \mathrm{m}$ de diámetro Cuatrecasasiella argentina

11'. Ora variable, si circular $>3 \mu \mathrm{m}$ de diámetro. 


\section{DISCUSIÓN}

Estos avances amplían el conocimiento morfológico de tipos polínicos característicos de la vegetación local de las turberas que poseen una gran diversidad de familias, géneros y especies (Hails 1996, Ramsar 2004) distribuidas a lo largo de los Andes centrales, y que aún no han sido descriptas en los atlas generales (Heusser 1971, Markgraf \& D’Antoni 1978, Graf 1979, Wingenroth \& Heusser 1984). Además son de considerable importancia en el campo de los estudios paleoecológicos, que utilizan, entre otras herramientas, las investigaciones palinológicas como base para la reconstrucción del paisaje. Estas contribuciones breves y específicas ayudarán, en la interpretación de los registros polínicos fósiles, a diferenciar la vegetación local de la extra regional, además de las situaciones ambientales azonales de disturbios naturales y antrópicos.

\section{AGRADECIMIENTOS}

Los autores agradecen a National Geographic Society (Grant \#8030-06), Bundesministerium für Bildung und Forschung (Projekt ARG 06/009), SeCTER-UNJu (Proyecto A 08/103) y Universität Trier por el financiamiento. A la Dra. Barbara Ruthsatz por sus valiosos aportes, al Lic. Fabio Flores por la asistencia técnica y a los revisores anónimos por las sugerencias para mejorar el manuscrito.

\section{BIBLIOGRAFÍA}

Braun Wilke, R.H. \& L.P.E. Picchetti. 1999. Deterioro de los Recursos Naturales en la Puna de Jujuy- Argentina. Agraria 1(4): 25-31.

Cabrera, A.L. 1976. Provincias fitogeográficas de Argentina. Enciclopedia Argentina de Agricultura y Jardinería. Ed. Acme. Bs. As. 85 pp.

Earle, L.R., B.G. Warner \& R. Aravena. 2003. Rapid development of an unusual peat-accumulating ecosystem in the Chilean Altiplano. Quaternary Research 59: 2-11.

Erdtman, G. 1957. Sobre la Terminología del Polen y las Esporas. Revista de la Facultad de Ciencias Agrarias, Universidad Nacional de Cuyo 6: 39-51.

Erdtman, G. 1960. The acetolysis method. Svensk Botanisk Tidskrift Utgifven af Svenska Botaniska Foreningen.
Stockholm 54: 561-564.

FAegri, K. \& J. Iversen. 1989. Textbook of Pollen Analysis. IV Edition. Blackburn Press, Caldwell. 328 pp.

Graf, K. 1979. Untersuchungen zur rezenten Pollen- und Sporenflora in der nördlichen Zentralkordilliere Boliviens und Versuch einer Auswertung von Profilen aus postglazialen Torfmooren. Habilitation. Universität Zürich. 104 pp.

Graf, K. 1992. Physische Geographie. Pollendiagramme aus den Anden. Eine Synthese zur Klimageschichte und Vegetationsentwicklung seit der letzten Eiszeit. Vol. 34. $138 \mathrm{pp}$.

Hails, A.J. (ed.). 1996. Wetlands, Biodiversity and the Ramsar Convention: The role of the convention on wetlands in the conservation and wise use of biodiversity. Ramsar Convention Bureau. Switzerland. 196 pp.

Heusser, C.J. 1971. Pollen and Spores of Chile. The University of Arizona Press. Tucson. 167 pp.

Markgraf, V. \& H. D'antoni. 1978. Pollen Flora of Argentina. The University of Arizona Press. Tucson. 208 pp.

Moore, P.D., J. A. Ween \& M. E. Collinson. 1991. Pollen Analysis. Blackwell Scientific Publications. Oxford. 216 pp.

Punt, W., P. P. Hoen, S. Blackmore, S. Nilsson \& L.E. Thomas. 2007. Glossary of pollen spore terminology. Review of Palaeobotany and Palynology 143: 1-81.

Ramsar. 2004. Manual de la Convención de Ramsar: Guía a la Convención sobre los Humedales (Ramsar, Irán, 1971), $4^{\mathrm{a}}$ ed. Ramsar Convention Bureau. Switzerland. 124 pp.

Ruthsatz, B. 2008. Hartpolstermoore der Hochanden NWArgentiniens als Indikatoren für Klimagradienten. In: J. Dengler, C. Dolnik \& M. Trepel (eds.), Flora, Vegetation und Naturschutz zwischen Schleswig-Holstein und Südamerika. - Festschrift für Klaus Dierßen zum 60. Geburtstag.Mitteilungen der Arbeitsgemeinschaft für Geobotanik in Schleswig-Holstein und Hamburg 65: 209-238.

Squeo, F.A., B.G. Warner, R. Aravena \& D. Espinoza. 2006. Bofedales: high altitude peatlands of the central Andes. Revista Chilena de Historia Natural. 79: 245-255.

Torres, G.R., K. Schittek, L.C. Lupo \& F.F. Flores. 2007. Situación ambiental del las turberas altoandinas en los Andes del Noroeste argentino. $2^{\circ}$ Foro Latino Americano de Montañas. Ed UNJu. Jujuy. Argentina. Pp: 53-54.

Wingenroth, M. \& C. J. Heusser. 1984. Polen de la alta cordillera. Quebrada Benjamín Matienzo. IANINGLA. Mendoza. Argentina. 195 pp.

Zuloaga, F.O., O. Morrone \& M.J. Belgrano (eds.). 2008. Catálogo de las Plantas Vasculares del Cono Sur. Monographs in Systematic Botany from the Missouri Botanical Garden, Volume 107. Missouri Botanical Garden. 3468 pp.

Recibido: 13.10 .11

Aceptado: 18.01 .12 